\title{
Risk factors associated with disease progression and mortality in chronic kidney disease of uncertain etiology: a cohort study in Medawachchiya, Sri Lanka
}

\author{
Lalantha Senevirathna $\cdot$ Tilak Abeysekera $\cdot$ Shanika Nanayakkara \\ Rohana Chandrajith - Neelakanthi Ratnatunga $\cdot$ Kouji H. Harada • \\ Toshiaki Hitomi $\cdot$ Toshiyuki Komiya $\cdot$ Eri Muso $\cdot$ Akio Koizumi
}

Received: 11 July 2011/ Accepted: 8 August 2011/Published online: 1 September 2011

(C) The Japanese Society for Hygiene 2011

\begin{abstract}
Background The alarming rise in the prevalence of chronic kidney disease of uncertain etiology ( $\mathrm{CKDu}$ ) among the low socioeconomic farming community in the North Central Province of Sri Lanka has been recognized as an emerging public health issue in the country.
\end{abstract}

For the Chronic Kidney Disease of Uncertain Aetiology Consortium.

A full list of Consortium members is provided in additional file 1 in the electronic supplementary material.

L. Senevirathna, T. Abeysekera, and S. Nanayakkara contributed equally to this work.

Electronic supplementary material The online version of this article (doi:10.1007/s12199-011-0237-7) contains supplementary material, which is available to authorized users.

\footnotetext{
L. Senevirathna $\cdot$ S. Nanayakkara $\cdot$ K. H. Harada $\cdot$

T. Hitomi · A. Koizumi $(\bowtie)$

Department of Health and Environmental Sciences,

Kyoto University Graduate School of Medicine,

Kyoto, Japan

e-mail: koizumi.akio.5v@kyoto-u.ac.jp

L. Senevirathna

e-mail: lalantha.s@kt3.ecs.kyoto-u.ac.jp

S. Nanayakkara

e-mail: shanika.n@at2.ecs.kyoto-u.ac.jp

K. H. Harada

e-mail: harada.koji.3w@kyoto-u.ac.jp

T. Hitomi

e-mail: hitomi.t@ax4.ecs.kyoto-u.ac.jp

T. Abeysekera

Nephrology Unit, General Hospital (Teaching),

Kandy, Sri Lanka

e-mail: tilak_1@hotmail.com
}

Methods This study sought to determine the possible factors associated with the progression and mortality of CKDu. The study utilized a single-center cohort registered in 2003 and followed up until 2009 in a regional clinic in the endemic region, and used a Cox proportional hazards model.

Results We repeatedly found an association between disease progression and hypertension. Men were at higher risk of CKDu than women. A significant proportion of the patients in this cohort were underweight, which emphasized the need for future studies on the nutritional status of these patients.

Conclusions Compared with findings in western countries and other regions of Asia, we identified hypertension as a major risk factor for progression of CKDu in this cohort.

Keywords Chronic kidney disease of uncertain etiology . Progression · Hypertension · Sri Lanka

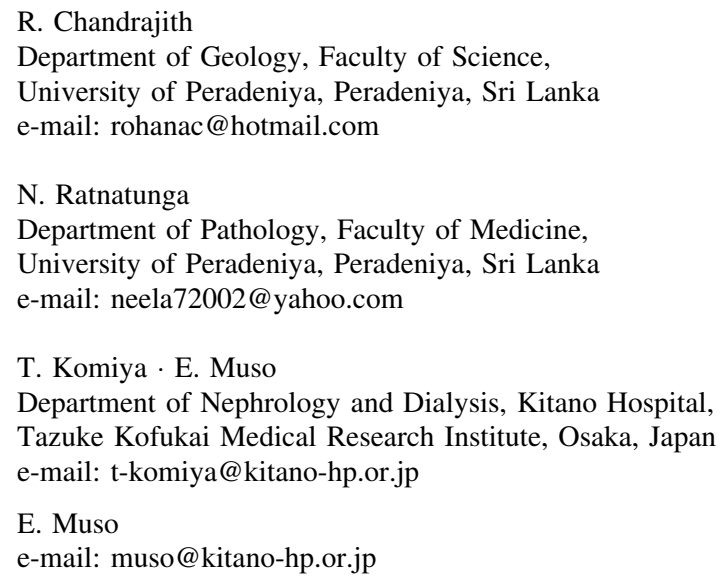




\section{Introduction}

Chronic kidney disease (CKD) has emerged as a global public health issue [1] and has become an important cause of morbidity and mortality [2]. Patients with CKD constitute a significant economic burden, both directly in terms of resource utilization, and indirectly through loss of productivity and impaired quality of life [3]. In the past decade, 1.1 trillion dollars have been spent on dialysis worldwide [4]. Therefore, early detection of asymptomatic CKD patients is important because early intervention has a reasonable chance of making a positive impact on outcome [5]. Similarly, it is important to prevent or delay disease progression from mild to severe stages [4]. The global increase in the prevalence of CKD and its disproportionate burden on economically developing countries is being driven by an increase in the prevalence of the main risk factors for CKD; namely, diabetes, hypertension, obesity, and aging of the population [6].

However, in the North Central Province (NCP) of Sri Lanka, the dramatically increasing prevalence of CKD during the past two decades has not been attributable to any of these factors [7]. Thus, it has been named CKD of uncertain etiology (CKDu). The majority of CKDu patients in the NCP are from the low socioeconomic farming community [8]. As a result of the insidious onset and progression of the disease, these patients present in the late stages, which requires renal replacement therapy such as dialysis and transplantation. In 2005, $4.6 \%$ of the national health budget (annual) was being spent on the management of renal disease patients [9].

In this CKDu endemic region, an epidemiological survey to evaluate the possible risk factors has revealed that a family history of CKDu, taking ayurvedic treatment, and a history of snake bite are significant predictors for $\mathrm{CKDu}$ occurrence [8]. As a result of the clinically observed heterogeneity in disease progression, we hypothesized that the rate of disease progression and death could be modified by different demographic, clinical, and lifestyle-related factors. To evaluate this, we followed up a CKDu cohort that was identified in a high-risk population screening program, which is believed to be the first-ever cohort study of CKDu in the NCP of Sri Lanka. It is expected that risk factor identification will be crucial in the prevention of CKDu progression from mild to severe stages.

\section{Methods}

Cohort

Target population screening programs using dipstick proteinuria were started in 2003 when there was a surge in new registrations with $\mathrm{CKDu}$ in the local renal clinics in the NCP. Early-morning urine samples from people aged $>5$ years (except for menstruating women) were checked on three occasions, with an interval of 1 week between consecutive samples. Subjects with proteinuria on two or more occasions were referred to renal clinics for further confirmatory investigations. Patients with $\mathrm{CKDu}$ were diagnosed after excluding known etiologies. Diabetes mellitus was excluded by the absence of a history, no current treatment, and hemoglobin A1C (HbA1C) $<6.5 \%$. Malignant hypertension was excluded by the absence of a history of chronic and/or severe hypertension. If blood pressure was $<160 / 100 \mathrm{mmHg}$ untreated or $<140 / 90 \mathrm{mmHg}$ with up to two antihypertensive agents, those people were included in the CKDu group. Systemic lupus erythematosus was excluded by the absence of anti-nuclear factor and double-stranded DNA antibody. In all cases, biopsy was conducted for pathological diagnosis. CKDu was pathologically defined as a region of focal-to-diffuse interstitial fibrosis and tubular atrophy. IgA nephropathy was excluded by immunostaining for $\operatorname{IgG}$, IgM, IgA, and complement component C3. Urological diseases of known etiology were excluded by clinical symptoms and routine investigations.

All patients who were diagnosed with $\mathrm{CKDu}$ were registered in local clinics. We recruited 143 CKDu patients at the time of registration at the Medawachchiya renal clinic after screening in 2003. All the patients recruited for the cohort were followed up for 72 months from registration until 2009 or death.

\section{Baseline risk factor assessment}

After registering the patients in the clinic, demographic and lifestyle-related information was collected by physicians through face-to-face interviews using a structured questionnaire. If available, medical records were used to collect the relevant clinical information. The risk factors of interest were: source of drinking water (agro-well, tube well, or garden well); habits (alcohol consumption, smoking, or betel chewing); family history of CKD, and history of snake bites; frequently used medication; and parental consanguinity. A history of hypertension, malaria, or bed-wetting, and ultrasonographic findings of shrunken kidney were collected from medical records. The presence of dental fluorosis was recorded after examination. Patients with mild hypertension (blood pressure $<160 / 100 \mathrm{mmHg}$ untreated, or $<140 / 90 \mathrm{mmHg}$ with up to two antihypertensive agents) were classified as hypertensive patients. If patients developed diabetes mellitus during the follow-up period, they were classified as diabetics. 


\section{Covariates}

\section{Body mass index}

The height and weight of the patients were measured in increments of $0.1 \mathrm{~cm}$ and $0.1 \mathrm{~kg}$, respectively, while they were wearing light clothing. Body mass index (BMI) was calculated as body weight $(\mathrm{kg})$ divided by the square of height $(\mathrm{m})$. BMI was categorized according to the World Health Organization guidelines: $<18.5 \mathrm{~kg} / \mathrm{m}^{2}$ (underweight), $18.5-24.9 \mathrm{~kg} / \mathrm{m}^{2}$ (normal), $25-29.9 \mathrm{~kg} / \mathrm{m}^{2}$ (overweight), and $\geq 30 \mathrm{~kg} / \mathrm{m}^{2}$ (obese).

\section{Serum creatinine and glomerular filtration rate}

The latest serum creatinine level was used to calculate the estimated glomerular filtration rate (eGFR) using the Modified Diet in Renal Disease (MDRD) formula [10]:

$$
\begin{aligned}
& \text { GFR }\left(\mathrm{ml} / \mathrm{min} / 1.73 \mathrm{~m}^{2}\right) \\
& =186 \times(\text { serum creatinine })^{-1.154} \times(\text { Age })^{-0.203} \\
& \quad \times(0.742 \text { if female }) \times(1.233) .
\end{aligned}
$$

According to one of the most widely adopted classifications, proposed by the Kidney Disease Outcomes Quality Initiative, five stages were defined for $\mathrm{CKDu}$ using the eGFR value [11]. If eGFR was $\geq 90 \mathrm{ml} / \mathrm{min} / 1.73 \mathrm{~m}^{2}$, but there was evidence of kidney damage such as proteinuria, patients were classified as CKDu stage 1. Stages 2-5 were classified according to eGFR values of 60-89, 30-59, $15-29$, and $<15 \mathrm{ml} / \mathrm{min} / 1.73 \mathrm{~m}^{2}$ in stages $2,3,4$, and 5 , respectively.

\section{Kidney disease follow-up}

For each member of the cohort, the person-years of followup were calculated from 2003 to the date of death or to the final year of the study period (2009). The entire cohort was closely monitored for 2 years (cohort established in 2003 and followed up in 2004 and 2005) for deaths and disease progression, and then the patients were invited to participate in the annual clinic to determine disease progression. It was observed that 34 patients were missing in 2005 without any records, and 81 were reported in 2006 and 76 in 2007. Forty-four patients were reported in 2008, and in 2009 only 25 were reported to the annual follow-up program.

\section{Statistical analysis}

Values for demographic variables, BMI, and history of hypertension in the study participants are presented as means with standard deviation (SD). MDRD-based eGFR was categorized by sex and various age groups, using the SAS Statistical Package version 9.1 (SAS Institute, Cary, NC, USA). After stratification by age and eGFR, sex/age adjusted hazard ratios (HRs) for $\mathrm{CKDu}$ were obtained using a Cox proportional hazards model. The risk factors considered in the analysis were: source of drinking water (agro-well, tube well, or garden well); habits (alcohol consumption, smoking, or betel chewing); family history of CKD; history of hypertension, malaria, bed-wetting, or snake bites; frequently used medication; parental consanguinity; and clinical findings (dental fluorosis, ultrasonographic findings of shrunken kidney, BMI, serum creatinine levels, and CKDu stage at follow-up). In the first trial of the Cox model, each factor was individually considered (univariate Cox model), and age-corrected, sexstratified significant factors for disease progression were identified for CKDu stage 1-4 patients, using SAS (PROC PHREG - outcome of the model was disease progression). Disease progression was determined according to the advance of CKDu stage over the monitoring period. In the second trial of the Cox model, all significant factors identified in the first analysis were combined (multivariate Cox model). Similar analysis was carried out for CKDu stage 1 and stage 2-3 patients at the third and fourth Cox model analyses for disease progression. Univariate and multivariate Cox model analyses (fifth and sixth Cox model analyses) were carried out to determine individual and combined mortality factors. A Kaplan-Meier curve was generated with SAS soft ware. Log-rank and Wilcoxon tests were carried out to check the homogeneity of the survival curves. All analyses were performed with the SAS Statistical Package version 9.1 (SAS Institute). This was a fact-finding study; therefore, we placed an emphasis on false-negative results. Thus, we considered $p<0.10$ as significant.

\section{Results}

Study cohort

The study cohort consisted of 143 patients with a mean age (SD) of 43 (6.5) years. They were predominantly Sinhalese in ethnicity $(86 \%)$ and belonged to rural communities. The number of participants and their demographic characteristics, BMI, and history of hypertension are shown in Table 1.

Fifty-eight percent of the participants were men. Importantly, the majority of the participants had low BMI (42\%) and only $8 \%$ had high BMI (overweight and obese). The prevalence of hypertension was $24 \%$, and $3 \%$ developed diabetes during the follow-up period. The distribution of MDRD-estimated GFR categories by sex and age is 
Table 1 Distribution of demographic variables, BMI, and history of hypertension among study participants in 2003

\begin{tabular}{lll}
\hline All participants & Number $(\%)$ & $\begin{array}{l}\text { Mean age (SD), } \\
\text { years }\end{array}$ \\
\hline Women & $60(42)$ & $38.42(20.66)$ \\
Men & $83(58)$ & $47.66(18.83)$ \\
Age (years) & & \\
$<20$ & $24(17)$ & $13.6(4.0)$ \\
$20-29$ & $16(11)$ & $23.6(2.9)$ \\
$30-39$ & $13(9)$ & $35.0(3.3)$ \\
$40-49$ & $26(18)$ & $43.8(3.0)$ \\
$50-59$ & $30(21)$ & $53.8(2.6)$ \\
$60-69$ & $20(14)$ & $64.0(2.0)$ \\
$>70$ & $14(10)$ & $76.6(6.5)$ \\
BMI $\left(\mathrm{kg} / \mathrm{m}^{2}\right)$ & & $46.3(23.0)$ \\
$<18.5$ & $52(42)$ & $44.4(16.6)$ \\
$18.5-24.9$ & $62(50)$ & $42.9(11.5)$ \\
$25-29.9$ & $8(6)$ & $31.0(1.4)$ \\
$\geq 30$ & $2(2)$ & $54.1(15.1)$ \\
Hypertension & $34(24)$ & \\
\hline
\end{tabular}

$B M I$ body mass index $\left(\mathrm{kg} / \mathrm{m}^{2}\right), S D$ standard deviation

presented in Table 2. The proportion of early-stage CKDu patients was markedly reduced in the older age groups. For example, in the youngest population (age $<20$ years), $92 \%$ were CKDu stage $1\left(\right.$ GFR $\left.>90 \mathrm{ml} / \mathrm{min} / 1.73 \mathrm{~m}^{2}\right)$, but in the older population (aged 60-69 years), the CKDu stage-1 population was only $9 \%$. In contrast, very few or no latestage patients were found in the younger age group. For example, no patients were found in CKDu stage 4 (GFR $15-29 \mathrm{ml} / \mathrm{min} / 1.73 \mathrm{~m}^{2}$ ) in the younger age group $(<20$ years), but almost $40 \%$ of the patients in the older age group (60-69 years) were CKDu stage 4.

The cohort was closely monitored for 2 years for disease progression and death, and they were invited to join the annual monitoring program for another 4 years. The mean period of observation was 39.9 months. It was found that 11 patients died during the monitoring period of 2003-2005, and the numbers of patients who came to the follow-up clinics in 2005, 2006, 2007, 2008, and 2009 were $90,81,76,44$, and 25 , respectively. Table 3 shows the number of deaths recorded during the first 2 years, categorized by $\mathrm{CKDu}$ stage and age (above and below 65 years). The mortality rates in the present study were compared with those reported in similar CKD cohort studies from different parts of the world.

Predictors of disease progression

A sex-stratified, age-adjusted, Cox proportional hazards model was used to identify the individual parameters for disease progression: occupation, drinking water source, alcohol consumption, smoking, betel chewing, family history, hypertension, diabetes mellitus, malaria, bed-wetting, snake bites, medication used, parental consanguinity, dental fluorosis, shrunken kidney, and BMI. Table 4 shows the significant factors obtained for disease progression for patients with stage 1-4 CKDu. Alcohol consumption, betel chewing, nonsteroidal anti-inflammatory drugs (NSAIDs), and hypertension were identified as major individual factors (age-adjusted and sex-stratified) for disease progression, with HRs of $3.39,3.61,2.33$, and 2.02, respectively $(p<0.1)$. In the multivariate analysis with all significant factors, alcohol consumption and hypertension were the main factors for disease progression, with HRs of 3.64 $(p=0.076)$ and $3.38(p=0.011)$, respectively. We repeated the same analysis for stage 1 and stage 2-3 patients, and the significant results are shown in Table 5. Hypertension was the only factor identified for disease progression in stage 1 patients, with an HR of $7.29(p=0.34)$. Even though it was statistically not significant $(p=0.12)$, it is worth highlighting the factor of family history, with an HR of 21.63. Hypertension, BMI, and malaria were identified as the significant factors for disease progression in stage 2 and 3 patients (sex-stratified and age-adjusted). Hypertension showed the highest HR, of $3.07(p=0.060)$, in this analysis. The dominance of hypertension was further emphasized by multivariate analysis, with an HR of 26.14 ( $p=0.003$ ); BMI and malaria showed lower HRs, of 0.64 $(p=0.011)$ and $0.27(0.083)$, respectively.

It was observed that hypertension repeatedly emerged as an important factor for disease progression, and we developed Kaplan-Meier survival curves to illustrate this further (Fig. 1). Different rank statistics tests were used to check the homogeneity of the survival curves. Log-rank and Wilcoxon tests showed $\chi^{2}$ values of $11.4(p=0.0007)$ and $10.1(p=0.0014)$, respectively, which suggested a comparatively rapid disease progression if the patients had a history of hypertension in the early stages of the disease.

In addition to hypertension, alcohol consumption and betel chewing were also identified as significant factors for disease progression by univariate Cox model analysis, but in the multivariate analysis, their significance disappeared. However, it is noteworthy that in a previous study, by Chou et al. [12], it was found that betel-nut use was associated with CKD in men and this association was independent of age, BMI, smoking, alcohol consumption, hypertension, diabetes, and hyperlipidemia.

Predictors of mortality

Univariate Cox survival analysis (age-adjusted and sexstratified) suggested that hypertension, $\mathrm{CKDu}$ stage, and shrunken kidney were the significant factors associated with mortality rate, with HRs of $2.29(p=0.1), 3.00$ 
Table 2 MDRD-based GFR according to sex and age in 2003

\begin{tabular}{|c|c|c|c|c|c|c|c|}
\hline & \multirow[t]{2}{*}{ Mean age (SD), years } & \multirow[t]{2}{*}{ Total no. } & \multicolumn{5}{|c|}{$\operatorname{GFR}\left(\mathrm{ml} / \mathrm{min} / 1.73 \mathrm{~m}^{2}\right)$} \\
\hline & & & $\begin{array}{l}>90 \\
\text { Stage } 1\end{array}$ & $\begin{array}{l}60-89 \\
\text { Stage } 2\end{array}$ & $\begin{array}{l}30-59 \\
\text { Stage } 3\end{array}$ & $\begin{array}{l}15-29 \\
\text { Stage } 4\end{array}$ & $\begin{array}{l}<15 \\
\text { Stage } 5\end{array}$ \\
\hline \multicolumn{8}{|c|}{ All participants } \\
\hline Women & $38.42(20.66)$ & 60 & $24(40.00)$ & $11(18.33)$ & $14(23.33)$ & $9(15.00)$ & $2(3.33)$ \\
\hline Men & $47.66(18.83)$ & 83 & $26(31.33)$ & $11(13.25)$ & $18(21.69)$ & $14(16.87)$ & $14(16.87)$ \\
\hline \multicolumn{8}{|c|}{ Age (years) } \\
\hline$<20$ & $13.6(4.0)$ & 26 & $24(92.30)$ & $0(0)$ & $1(3.87)$ & $0(0)$ & $1(3.83)$ \\
\hline $20-29$ & $23.6(2.9)$ & 16 & $9(56.25)$ & $5(31.25)$ & $2(12.50)$ & $0(0)$ & $0(0)$ \\
\hline $30-39$ & $35.0(3.3)$ & 14 & $4(28.57)$ & $4(28.57)$ & $3(21.43)$ & $1(7.14)$ & $2(14.29)$ \\
\hline $40-49$ & $43.8(3.0)$ & 27 & $7(25.93)$ & $6(22.22)$ & $5(18.52)$ & $7(25.93)$ & $2(7.41)$ \\
\hline $50-59$ & $53.8(2.6)$ & 28 & $4(14.29)$ & $3(10.17)$ & $12(42.86)$ & $4(14.29)$ & $5(17.86)$ \\
\hline $60-69$ & $64.0(2.0)$ & 22 & $2(9.09)$ & $2(9.09)$ & $5(22.73)$ & $9(40.91)$ & $4(18.18)$ \\
\hline$>70$ & $76.6(6.5)$ & 10 & $0(0)$ & $2(20.00)$ & $4(40.00)$ & $2(20)$ & $2(20)$ \\
\hline
\end{tabular}

Values are numbers (percentages) of participants unless stated otherwise

GFR glomerular filtration rate $\left(\mathrm{ml} / \mathrm{min} / 1.73 \mathrm{~m}^{2}\right), M D R D$ Modification of Diet in Renal Disease, $S D$ standard deviation

Table 3 CKDu cohort deaths during first 2 years follow-up (2004 and 2005)

\begin{tabular}{llcl}
\hline Age group (years) & CKD stage & Number $(\%)$ & Observed deaths \\
\hline$<65$ & 1 and 2 & $51(53.13)$ & 0 \\
& 3 & $20(20.83)$ & 1 \\
& 4 & $16(16.67)$ & 3 \\
$>65$ & 5 & $9(9.34)$ & 4 \\
& Total & 96 & 8 \\
& 1 and 2 & $4(30.77)$ & 0 \\
& 3 & $4(30.77)$ & 0 \\
& 4 & $2(15.38)$ & 1 \\
& 5 & $3(23.08)$ & 2 \\
& Total & 13 & 3
\end{tabular}

Values are numbers (percentages) of participants unless stated otherwise

$C K D u$ chronic kidney disease of uncertain etiology

( $p=0.00)$, and $2.74(p=0.09)$, respectively (Table 4$)$. In multivariate Cox survival analysis, the dominance of CKDu stage was observed over other significant factors, with an HR of $2.94(p=0.001)$.

\section{Discussion}

This study is believed to be the first to identify possible risk factors of CKDu disease progression and death in a prospective cohort in Madawachchiya, Sri Lanka, where $\mathrm{CKDu}$ is endemic.

The age-structure pyramid of this CKDu cohort was broad-based, with a relatively large proportion of younger patients. For example, $17 \%$ of the $\mathrm{CKDu}$ patients were
$<20$ years old. In contrast, the proportion of CKD patients aged $<20$ years in the United States is $<2 \%$ [13]. Almost all the younger patients $(<20$ years $)$ in this cohort had $\mathrm{CKDu}$ stage 1 and they comprised $24 \%$ of the stage- 1 population. Therefore, it is reasonable to presume that the problem will become much more serious in the coming few decades when these patients progress to late stages of the disease. Even though the older age group ( $>70$ years) comprises $37 \%$ of the CKD population in the United States [13], only about $10 \%$ of the patients in our cohort were $>70$ years old.

A similar age-structure pyramid in the CKD population has been reported from another Asian country, Iran, where $23.6 \%$ are aged $<30$ years, and only $6.0 \%$ are $>70$ years old [4]. In the present cohort, $37 \%$ of stage $3-5 \mathrm{CKDu}$ patients were older than 60 years.

In our cohort, the probability of death in a 2-year period was $23 \%$ for a patient older than 65 years and that was $8 \%$ for a patient younger than 66 years. These values are almost double those reported by Manns et al. [14], who showed that the probability of death in a CKD cohort in Alberta, Canada, was $4.81 \%$ for patients aged $<66$ years and $13.29 \%$ for those aged $>65$ years. It is particularly interesting to note that $81 \%$ of the Canadian cohort had CKD stage 1 or $2,18 \%$ had stage 3 , and $1 \%$ had stage 4 or 5 , whereas in our cohort, $50 \%$ had stage 1 or $2,22 \%$ had stage 3 , and $28 \%$ had stage 4 or 5 . This demonstrated that the disease progression in our CKDu cohort was much faster than that in western countries, and we suspect that this could be a reason for the higher death rate in our cohort. The overall death rate in our cohort was $5.3 \%$ per 100 patient-years, which is higher than the rate reported in Taiwan [15]. Lack of adequate dialysis facilities to manage 
Table 4 Sex-stratified, age-adjusted univariate and multivariate Cox proportional models for CKDu disease progression and death

\begin{tabular}{|c|c|c|c|c|c|}
\hline Outcome & Type of analysis & Variable & $\begin{array}{l}\text { Hazard } \\
\text { ratio }\end{array}$ & $\begin{array}{l}95 \% \text { Confidence } \\
\text { interval }\end{array}$ & $p$ value \\
\hline \multirow{8}{*}{$\begin{array}{l}\text { Disease progression } \\
\quad(\text { CKD stage } 1-4)\end{array}$} & \multirow[t]{4}{*}{ Sex-stratified, age-adjusted univariate analysis } & Alcohol & 3.39 & $1.43-8.04$ & 0.006 \\
\hline & & Betel & 3.61 & $1.21-10.77$ & 0.021 \\
\hline & & Hypertension & 2.02 & $0.09-45.34$ & 0.907 \\
\hline & & NSAID & 2.33 & $0.95-5.71$ & 0.065 \\
\hline & \multirow{4}{*}{$\begin{array}{l}\text { Sex-stratified, age-adjusted multivariate } \\
\text { (alcohol, betel, hypertension, NSAID) } \\
\text { analysis }\end{array}$} & Alcohol & 3.64 & $0.87-15.23$ & 0.076 \\
\hline & & Betel & 1.94 & $0.52-7.24$ & 0.325 \\
\hline & & Hypertension & 3.38 & $1.31-8.72$ & 0.011 \\
\hline & & NSAID & 1.28 & $0.42-3.90$ & 0.662 \\
\hline \multirow[t]{6}{*}{ Death (CKD stage 1-5) } & \multirow[t]{3}{*}{ Sex-stratified, age-adjusted univariate analysis } & Hypertension & 2.29 & $0.85-6.17$ & 0.100 \\
\hline & & CKD stage & 3.00 & $1.71-5.26$ & 0.000 \\
\hline & & Shrunken kidney & 2.74 & $0.83-9.05$ & 0.097 \\
\hline & \multirow{3}{*}{$\begin{array}{l}\text { Sex-stratified, age-adjusted multivariate } \\
\text { (hypertension, CKD stage, shrunken kidney) } \\
\text { analysis }\end{array}$} & Hypertension & 1.79 & $0.62-5.17$ & 0.277 \\
\hline & & CKD stage & 2.94 & $1.57-5.51$ & 0.001 \\
\hline & & Shrunken kidney & 0.80 & $0.21-3.05$ & 0.742 \\
\hline
\end{tabular}

NSAID nonsteroidal anti-inflammatory drug, CKDu chronic kidney disease of uncertain etiology, Betel betel chewing, Alcohol alcohol consumption

Table 5 Sex-stratified, age-adjusted univariate and multivariate Cox proportional models of disease progression for stage-specific analysis

\begin{tabular}{|c|c|c|c|c|c|}
\hline $\mathrm{CKDu}$ & Type of analysis & Variable & Hazard ratio & $\begin{array}{l}95 \% \text { Confidence } \\
\text { interval }\end{array}$ & $p$ value \\
\hline \multirow[t]{2}{*}{ Stage 1} & \multirow[t]{2}{*}{ Sex-stratified, age-adjusted univariate analysis } & Hypertension & 7.29 & $1.16-45.81$ & 0.034 \\
\hline & & Family history & 21.63 & $0.44-1063.31$ & 0.120 \\
\hline \multirow[t]{6}{*}{ Stage $2-3$} & \multirow[t]{3}{*}{ Sex-stratified, age-adjusted univariate analysis } & Hypertension & 3.07 & $0.95-9.92$ & 0.060 \\
\hline & & BMI & 0.85 & $0.72-1.00$ & 0.070 \\
\hline & & Malaria & 0.26 & $0.08-0.85$ & 0.027 \\
\hline & \multirow{3}{*}{$\begin{array}{l}\text { Sex-stratified, age-adjusted multivariate (hypertension, } \\
\text { BMI, malaria) analysis }\end{array}$} & Hypertension & 26.14 & $3.04-224.77$ & 0.003 \\
\hline & & BMI & 0.64 & $0.45-0.91$ & 0.011 \\
\hline & & Malaria & 0.27 & $0.06-1.22$ & 0.083 \\
\hline
\end{tabular}

$B M I$ body mass index $\left(\mathrm{kg} / \mathrm{m}^{2}\right), C K D u$ chronic kidney disease of uncertain etiology

the CKDu patients in this region might have contributed to this higher mortality.

BMI is associated with CKD [16, 17]. Many researchers have reported that higher BMI is a risk factor for CKD in apparently healthy persons $[18,19]$. By contrast, in our cohort, only $8 \%$ of the patients were overweight $\left(25-29.9 \mathrm{~kg} / \mathrm{m}^{2}\right)$ or obese $\left(>30 \mathrm{~kg} / \mathrm{m}^{2}\right)$, whereas $42 \%$ of the patients were underweight $\left(<18.5 \mathrm{~kg} / \mathrm{m}^{2}\right)$. According to some studies, malnutrition has been shown to increase mortality risk more than high BMI [20, 21], and malnutrition could have also contributed to the higher death rate in our cohort.

In our study, men (58\%) were at higher risk of CKDu than women were $(42 \%)$. A similar association has been reported in a study in Taiwan [15], where $62 \%$ of the affected population was male. However, the majority of published studies have shown female-dominant CKD cohorts $[4,22,23]$. Further research is needed to determine the reasons for male dominance in our study area. However, factors such as genetic susceptibility and some behavioral risk factors, such as alcohol consumption and betel chewing, which could play a role in increasing disease occurrence, should not be neglected.

Diabetes and hypertension are known risk factors for CKD [24, 25]. According to the definition of CKDu, patients with diabetes and uncontrollable hypertension were not included in the present cohort. However, it should be noted that five patients developed diabetes and several developed hypertension during the follow-up period. At the same time, several patients had mild hypertension at the 


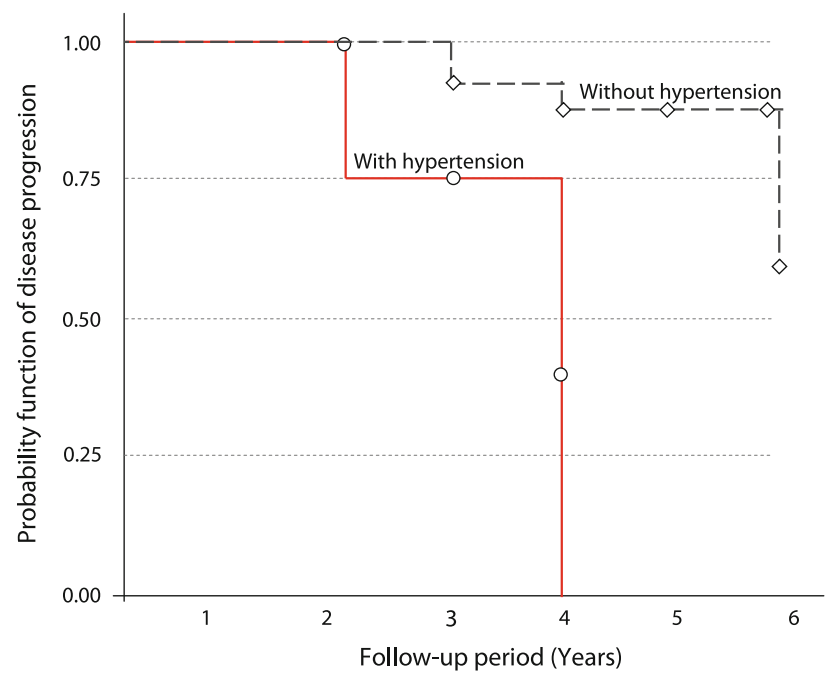

Fig. 1 Kaplan-Meier curves showing the probability of chronic kidney disease of uncertain etiology $(\mathrm{CKDu})$ disease progression over the monitoring period with and without self-reported hypertension for CKDu stage 1 (glomerular filtration rate [GFR] $>90 \mathrm{ml} / \mathrm{min} / 1.73 \mathrm{~m}^{2}$ ) patients (please refer to the "Results" section in the text for Log-rank test and Wilcoxon test results)

time of diagnosis of CKDu. Among the factors that were evaluated as suspected risk factors for disease progression in our cohort, hypertension was identified as the leading determinant of disease progression. Self-reported hypertension was a significant predictor $(p<0.05)$ for disease progression, with an HR of 3.38 by multivariate Cox proportional model analysis for stage 1-4 patients. Our results suggest a strong correlation between hypertension in the early stage of CKDu and disease progression. Sixty-six percent of stage 1 patients in our cohort were from the population aged $<30$ years, and hypertension showed an HR of 7.26 in a sex-stratified, age-adjusted Cox proportional model for stage 1 patients. A similar correlation was observed for stage 2-3 patients, with an HR of 26.16. Many researchers have shown that the prevalence of hypertension in South Asian populations is higher than that in western populations [26]. These results strongly suggest that tight control of blood pressure, particularly in the early stage of $\mathrm{CKDu}$, has a beneficial effect to slow down disease progression.

CKDu stage was the only factor identified to have a correlation with death, with HRs of 3.0 and 2.94 by univariate (sex-stratified and age-adjusted) and multivariate Cox proportional model analysis, respectively. This result is supported by the fact that almost all reported deaths were in patients with stage 4 or 5 disease. In contrast, many studies have reported that CKD patients in western countries frequently die before developing end-stage CKD [2729]. The reason for this could be the difference in age at disease initiation, because in many countries, CKD is common in the older age groups [30]. In the elderly population, eGFR is reduced in the normal aging process, but elderly patients have a greater mortality risk when compared with younger patients with the same eGFR range [30]. Therefore, it is likely that elderly patients will die in the middle of the disease process rather than reaching the end stage of CKD. However, our CKDu cohort was relatively younger than the cohorts that have been studied in other countries $[15,24,25]$. Thus, we can imagine that these CKDu patients would survive until they reach endstage CKD, in contrast to the population who are already in the latter part of their life when they begin the disease process.

Our study had a number of limitations. The study was started at the initiation of community screening in this endemic area; therefore, we could recruit only a limited number of patients. We used the MDRD formula for estimation for GFR, which has not been validated for the Sri Lankan population and across the age range. Therefore, the use of eGFR for staging the patients might not have reflected the actual GFR for this population. For the exclusion of diabetes mellitus, we used a cut-off HbA1C level of $6.5 \%$. However, previous studies have shown that such a cut-off level misses a substantial number of people with type 2 diabetes, including some with fasting hyperglycemia, as well as people with impaired glucose tolerance [31]. Therefore, there is a possibility that we might have recruited some diabetic patients in this cohort because of misdiagnosis.

\section{Conclusions}

In this study, we tried to determine factors possibly associated with progression and mortality of CKDu in a singlecenter cohort registered in the Medawachchiya regional clinic in Sri Lanka. We repeatedly found an association between disease progression and hypertension. Men were at higher risk of CKDu than women. A significant proportion of the patients in this cohort were underweight, indicating a need for future studies on the nutritional status of these patients. Compared with findings in western countries and other regions of Asia, younger age at disease onset, equal patient distribution in different age groups, faster disease progression in those with hypertension, deaths occurring mostly in end-stage $\mathrm{CKDu}$, and relatively higher mortality rate are some of the characteristics that were identified in this cohort.

Acknowledgments The authors would like to thank Dr. Arjuna Dandeniya, Dr. Gayan Wijesundara, and Dr. Amila Kodikara; the temporary lecturers of the Department of Pharmacology, Faculty of Medicine, University of Peradeniya, Sri Lanka, for their support in data handling. This study was supported by the Special Coordination 
Funds for Promoting Science and Technology from the Ministry of Education, Culture, Sports, Science and Technology in Japan. The funding agency had no role in the study design, data collection, and analysis, or in the decision to publish or in the preparation of the manuscript. TA, EM, and AK conceived and designed the experiments. TA and RC performed the experiments. STMLDS, SN, KHH and TH statistically analyzed the data. STMLDS, SN, KHH, and AK wrote the paper. NR, TK, EM, and AK critically revised the draft. All authors read and approved the manuscript.

Conflict of interest The authors declare that they have no competing interests.

\section{References}

1. Hwang SJ, Tsai JC, Chen HC. Epidemiology, impact and preventive care of chronic kidney disease in Taiwan. Nephrology (Carlton). 2010;15(2):3-9.

2. Perlman RL, Finkelstein FO, Liu L, Roys E, Kiser M, Eisele G, Burrows-Hudson S, Messana JM, Levin N, Rajagopalan S, Port FK, Wolfe RA, Saran R. Quality of life in Chronic Kidney Disease (CKD): a cross-sectional analysis in the Renal Research Institute-CKD study. Am J Kidney Dis. 2005;45(4):658-66.

3. Menon V, Sarnak MJ. The epidemiology of chronic kidney disease stages 1 to 4 and cardiovascular disease: a high-risk combination. Am J Kidney Dis. 2005;45(1):223-32.

4. Najafi I, Attari F, Islami F, Shakeri R, Malekzadeh F, Salahi R, Gharavi MY, Hosseini M, Broumand B, Haghighi AN, Larijani $\mathrm{B}$, Malekzadeh R. Renal function and risk factors of moderate to severe chronic kidney disease in Golestan Province, northeast of Iran. PLoS One. 2010;5(12):e14216.

5. Brown WW, Peters RM, Ohmit SE, Keane WF, Collins A, Chen SC, King K, Klag MJ, Molony DA, Flack JM. Early detection of kidney disease in community settings: the Kidney Early Evaluation Program (KEEP). Am J Kidney Dis. 2003;42:22-35.

6. Ayodele OE, Alebiosu CO. Burden of chronic kidney disease: an international perspective. Adv Chronic Kidney Dis. 2010;17(3): 215-24.

7. Chandrajith R, Nanayakkara S, Itai K, Aturaliya TN, Dissanayake CB, Abeysekara T, Harada K, Watanabe T, Koizumi A. Chronic kidney disease of uncertain aetiology (CKDue) in Sri Lanka: geographic distribution and environmental implications. Environ Geochem Health. 2011;409(11):671-5.

8. Wanigasuriya KP, Peiris-John RJ, Wickremasinghe R, Hittarage A. Chronic renal failure in North Central Province of Sri Lanka: an environmentally induced disease. Trans R Soc Trop Med Hyg. 2007;101:1013-7.

9. World Health Organization (WHO) country office Sri LankaNews letters. http://www.whosrilanka.org/EN/Section1_76.htm Accessed 10 Dec 2010.

10. Levey AS, Bosch JP, Lewis JB, Modification of Diet in Renal Disease Study Group. A more accurate method to estimate glomerular filtration rate from serum creatinine: a new prediction equation. Ann Intern Med. 1999;130:461-70.

11. The National Kidney Foundation. K/DOQI Clinical practice guidelines for chronic kidney disease: evaluation, classification, and stratification. Am J Kidney Dis. 2002;39:S46-75.

12. Chou CY, Cheng SY, Liu JH, Cheng WC, Kang IM, Tseng YH, Shih $\mathrm{CM}$, Chen W. Association between betel-nut chewing and chronic kidney disease in men. Public Health Nutr. 2009;12(5):723-7.
13. United States Renal Data System (USRDS) Annual data report 2010. http://www.usrds.org/

14. Manns B, Hemmelgarn B, Tonelli M, Au F, Hiasson C, Dong J, Klarenbach C. Population based screening for chronic kidney disease: cost effectiveness study. BMJ. 2010;341:c5869.

15. Chiu Y, Chien K, Lin S, Chen Y, Tsai T, Wu D. Outcomes of stage 3-5 chronic kidney disease before end-stage renal disease at a single center in Taiwan. Nephron Clin Pract. 2008;109: c109-18.

16. Nomura I, Kato J, Kitamura K. Association between body mass index and chronic kidney disease: a population-based, crosssectional study of a Japanese community. Vasc Health Risk Manag. 2009;5:315-20.

17. Chalmers L, Kaskel FJ, Bamgbola O. The role of obesity and its bioclinical correlates in the progression of chronic kidney disease. Adv Chronic Kidney Dis. 2006;13(4):352-64.

18. Hsu CY, McCulloch CE, Iribarren C, Darbinian J, Go AS. Body mass index and risk for end-stage renal disease. Ann Intern Med. 2006;144:21-8.

19. Gelber RP, Kurth T, Kausz AT, Manson JE, Buring JE, Levey AS, Gaziano JM. Association between body mass index and CKD in apparently healthy men. Am J Kidney Dis. 2005;46:871-80.

20. Beddhu S. The body mass index paradox and an obesity, inflammation, and atherosclerosis syndrome in chronic kidney disease. Semin Dialysis. 2004;17:229-32.

21. Kalantar-Zadeh K, Block G, Humphreys MH, Kopple JD. Reverse epidemiology of cardiovascular risk factors in maintenance dialysis patients. Kidney Int. 2003;63:793-808.

22. Zhang QL, Rothenbacher D. Prevalence of chronic kidney disease in population-based studies: systematic review. BMC Public Health. 2008;8:117.

23. Ong-Ajyooth L, Vareesangthip K, Khonputsa P, Aekplakorn W. Prevalence of chronic kidney disease in Thai adults: a national health survey. BMC Nephrol. 2009;10:35.

24. Coresh J, Astor BC, Greene T, Eknoyan G, Levey AS. Prevalence of chronic kidney disease and decreased kidney function in the adult US population: Third National Health and Nutrition Examination Survey. Am J Kidney Dis. 2003;41:1-12.

25. Chen J, Wildman RP, Gu D, Kusek JW, Spruill M. Prevalence of decreased kidney function in Chinese adults aged 35 to 74 years. Kidney Int. 2005;68:2837-45.

26. Jones CA, Mawani S, King KM, Allu SO, Smith M, Mohan S, Campbell NRC. Tackling health literacy: adaptation of public hypertension educational materials for an Indo-Asian population in Canada. BMC Public Health. 2011;11(1):24.

27. Adler AI, Stevens RJ, Manley SE, Bilous RW, Cull CA, Holman RR. Development and progression of nephropathy in type 2 diabetes: the United Kingdom Prospective Diabetes Study (UKPDS 64). Kidney Int. 2003;63:225-32.

28. Kovesdy CP, Trivedi BK, Anderson JE. Association of kidney function with mortality in patients with chronic kidney disease not yet on dialysis: a historical prospective cohort study. Adv Chronic Kidney Dis. 2006;13:183-8.

29. Go AS, Chertow GM, Fan D, McCulloch CE, Hsu CY. Chronic kidney disease and the risks of death, cardiovascular events, and hospitalization. N Engl J Med. 2004;351:1296-305.

30. O'Hare AM, Choi AI, Bertenthal D, Bacchetti P, Garg AX, Kaufman JS, Walter LC, Mehta KM, Steinman MA, Allon M, McClellan WM, Landefeld CS. Age affects outcomes in chronic kidney disease. J Am Soc Nephrol. 2007;18:2758-65.

31. Herman WH, Fajans SS. Hemoglobin A1c for the diagnosis of diabetes: practical considerations. Pol Arch Med Wewn. 2010;120(1-2):37-40. 